\title{
Cor triatriatum associado à insuficiência mitral e fibrilação atrial em adulto de 36 anos
}

\author{
Cor triatriatum with mitral insufficiency and atrial fibrillation in a 36-year-old man
}

Renato BRAULIO ${ }^{1}$, Cláudio Léo GELAPE², Carlos Camilo Smith FIGUEROA³ , Antônio Luiz Pinho RIBEIRO ${ }^{4}$

RBCCV 44205-899

\section{DADOS CLÍNICOS}

Adulto de 36 anos, sexo masculino, leucoderma, natural de Patos de Minas (MG), trabalhador rural. Paciente relatou que desde criança não conseguia praticar esportes ou fazer esforços moderados, pois apresentava fadiga e dispnéia. Há cerca de um ano, apresentou episódio de palpitação após esforço físico, seguido de dispnéia, dor torácica e lipotímia. Procurou pronto atendimento hospitalar, onde recebeu cardioversão elétrica, devido a fibrilação atrial com resposta alta e repercussão hemodinâmica. Recebeu alta em uso de losartam, furosemida e varfarina, mantendo-se em fibrilação atrial com freqüência de $80 \mathrm{bpm}$. Durante propedêutica posterior, o ecocardiograma revelou cor triatriatum associado à insuficiência mitral grave.

Com critérios ecocardiográficos para correção cirúrgica, além de alguns episódios posteriores de descompensação clínica, foi encaminhado para pré-operatório no Hospital das Clínicas da UFMG, onde chegou em classe funcional III (NYHA), há cerca de três meses da cirurgia.

Ao exame clínico, apresentava-se afebril, com bom estado geral e eupnéico em repouso; ausculta pulmonar sem ruídos adventícios; ausculta cardíaca com sopro sistólico regurgitativo em foco mitral com irradiação para axila (grau III/VI), ritmo irregular, FC: 76 bpm, PA: 110 x $80 \mathrm{mmHg}$, pulsos periféricos cheios; abdome livre com borda hepática a $2 \mathrm{~cm}$ do rebordo costal direito.

\section{ELETROCARDIOGRAMA}

Arrítmico com padrão de fibrilação atrial (FA), freqüência de $72 \mathrm{bpm}$, sobrecarga atrial esquerda e direita (SAE e D), sobrecarga ventricular esquerda e direita (SVE e D).

\section{RADIOGRAMA}

Área cardíaca aumentada, protusão de átrio direito, aumento da trama vascular pulmonar central com sinais de congestão leve.

1. Residência Médica - MEC; Cirurgião Cardiovascular.

2. Mestrado em Cirurgia, Doutorando em Cirurgia; Cirurgião Cardiovascular, Coordenador da Residência Médica em Cirurgia Cardiovascular do Hospital das Clínicas da UFMG.

3. Membro Titular da SBCCV, ex-professor da UFMG, Chefe dos Serviços de Cirurgia Cardiovascular do Hospital Felício Rocho e Hospital das Clínicas da UFMG.

4. Doutor em Medicina, Professor da UFMG, Chefe do Departamento de Cardiologia e Cirurgia Cardiovascular do Hospital das Clínicas da UFMG, Vice-diretor do Hospital das Clínicas da UFMG.

Trabalho realizado no Hospital das Clínicas da Universidade Federal de Minas Gerais (UFMG), Belo Horizonte, MG. 


\section{ECOCARDIOGRAMA}

Situs solitus em levocardia, conexões venoatriais e ventrículo-arteriais normais. Presença de membrana em átrio esquerdo com orifício de cerca de $1,5 \mathrm{~cm}$, insuficiência mitral grave, sem espessamento valvar, insuficiência tricúspide leve, hipocinesia difusa leve. Fração de ejeção: 54\%; ventrículo esquerdo (VE) na sístole: $47 \mathrm{~mm}$; VE na diástole: $66 \mathrm{~mm}$, átrio esquerdo: $51 \mathrm{~mm}$; pressão sistólica de artéria pulmonar (PSAP): $50 \mathrm{mmHg}$.

\section{DIAGNÓSTICO}

A descompensação clínica ocorrida há 1 ano, com fibrilação atrial e presença ao exame clínico de sopro regurgitativo em foco mitral, associada a dispnéia e fadiga aos médios esforços desde criança, levou a forte suspeita de doença cardíaca subjacente. Feito propedêutica, o ecocardiograma revelou, com clareza, a presença de insuficiência mitral grave pura sem sinais de calcificação ou estenose, presença de cor triatriatum, com membrana dividindo o átrio $\mathrm{E}$ em duas câmaras, com orifício central de cerca de $1,5 \mathrm{~cm}$ e a desembocadura das veias pulmonares na câmara proximal (não valvar). Além da boa visibilização anatômica nesse caso, o ECO nos forneceu dados precisos em relação às dimensões, pressões cavitárias e função miocárdica, compatíveis com a clínica do paciente.

\section{OPERAÇÃO}

Esternotomia mediana, instalação de circuito de circulação extracorpórea, heparinização sistêmica, colocação de cânulas de aorta e cavas (com cerclagem), hipotermia a $34^{\circ} \mathrm{C}$, cardioplegia cristalóide intermitente, anterógrada e hipotérmica a $4^{\circ} \mathrm{C}$. Após a abertura do átrio esquerdo, foi observada a presença de membrana com aspecto de diafragma [1], com orifício ovalado (Figura 1) de cerca de 1,5 $\mathrm{cm}$, permitindo a comunicação dessa parte atrial livre, por onde desembocava as quatro veias pulmonares, com a outra parte, onde se encontrava a valva mitral. Além disso, a valva mitral apresentava-se degenerada, muito frágil e insuficiente, sem espessamento. Feita a ressecção da membrana do cor triatriatum (Figura 2), a valva mitral foi examinada e optou-se por não ser submetida a plastia, haja vista o enorme grau de degeneração e fragilidade valvar. Realizada a troca valvar mitral por prótese mecânica. Realizada, ainda, ablação endocárdica transoperatória pela técnica de compartimentação atrial (MAZE) [2,3], primeiramente no átrio esquerdo e em seguida no átrio direito, usando cautério comum em baixa intensidade. O exame anatomopatológico da valva mitral revelou degeneração mixomatosa. O paciente recebeu alta no oitavo dia de pós-operatório, com anticoagulação plena, em ritmo sinusal com bloqueio atrioventricular de primeiro grau e em boas condições clínicas.

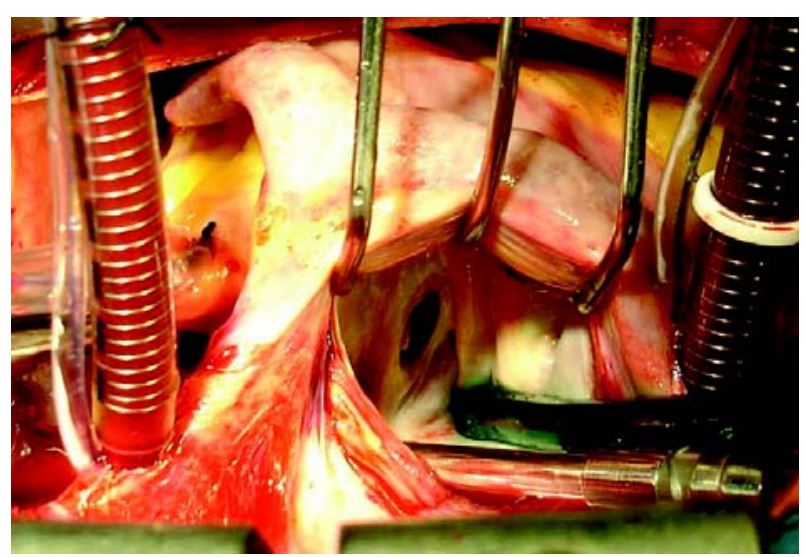

Fig. 1 - Átrio esquerdo aberto evidenciando a presença de membrana com orifício ovalado de cerca de $1,5 \mathrm{~cm}$, dividindo-o em duas câmaras comunicantes

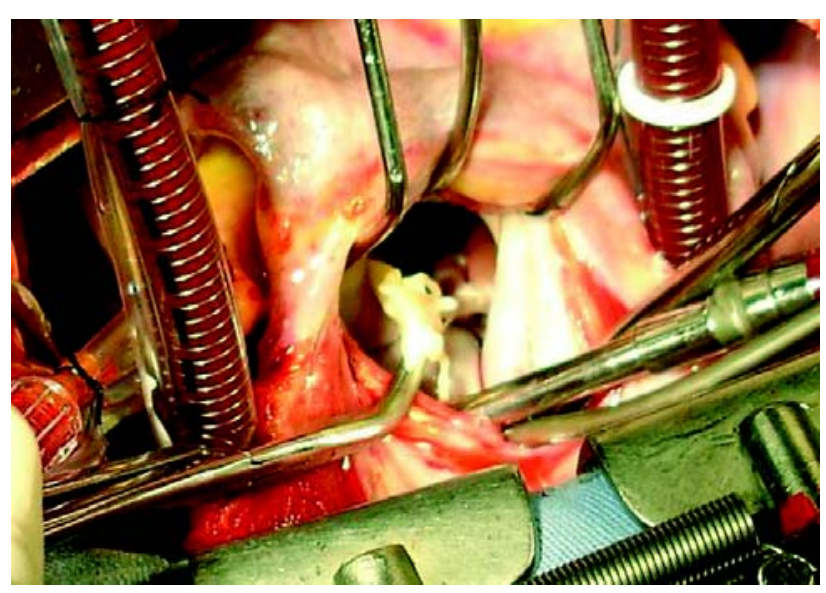

Fig. 2 - Ressecção da membrana do cor triatriatum, transformando o átrio esquerdo em uma câmara única

\section{REFERÊNCIAS}

1. Sugimori H, Noma M, Ikeda A, Tokunaga C, Imazuru T, Hiramatsu Y, et al. Cor triatriatum diagnosed in adulthood; report of a case. Kyobu Geka. 2005;58(10):893-5.

2. Brick AV, Seixas T, Portilho C, Peres AK, Vieira Jr JJ, Melo Neto R, et al. Tratamento intra-operatório da fibrilação atrial crônica com ultra-som. Rev Bras Cir Cardiovasc. 2001;16(4):337-49.

3. Nakajima H, Kobayashi J, Kurita T, Kitamura S. Maze procedure and cor triatriatum repair. Ann Thorac Surg. 2002;74(1):251-3. 